\title{
Role of lymphangiogenesis and lymphangiogenic factors during pancreatic cancer progression and lymphatic spread
}

\author{
MARTIN SCHNEIDER ${ }^{1}$, PETER BÜCHLER ${ }^{1}$, NATHALIA GIESE ${ }^{1}$, THOMAS GIESE ${ }^{1}$, \\ JÖRG WILTING ${ }^{2}$, MARKUS W. BÜCHLER ${ }^{1}$ and HELMUT FRIESS ${ }^{1}$ \\ ${ }^{1}$ Department of General Surgery, University of Heidelberg; ${ }^{2}$ Department of Pediatrics, University of Göttingen, Germany
}

Received May 27, 2005; Accepted July 4, 2005

\begin{abstract}
Treatment options for ductal adenocarcinoma of the pancreas are limited by early lymphatic spread, but the lymphatic vessels in pancreatic carcinoma have not been studied to date. Here, we present a histomorphological analysis of lymphatic vessels in pancreatic cancer resection specimens. Both intratumoral and peritumoral tissue were devoid of active lymphangiogenesis. Intratumoral lymphatics were frequently collapsed and non-functional, whereas peritumoral lymphatic vessels were enlarged, and numerous lymphatic vessels were seen in metastases. In addition, we screened pancreatic cancer tissue and pancreatic carcinoma cell lines for mRNA expression of the lymphangiogenic growth factor, VEGF-C; its receptor, VEGFR-3/flt4; and Prox1, a transcription factor essential for embryonic development of both lymphatic vessels and the pancreatic bud. VEGF-C was abundantly expressed in pancreatic cancer tissue and -cell lines and VEGFR-3/flt4 was expressed in cancer stromal cells. Prox 1 was strongly expressed in the normal exocrine pancreas but significantly reduced in pancreatic cancer specimens from patients with short survival rates. Well-differentiated cell lines displayed higher levels of Prox1 mRNA than poorly differentiated ones. These results suggest that active lymphangiogenesis is not required for lymphovascular spread of pancreatic cancer. VEGF-C may promote local tumor growth via paracrine signaling to stromal cells expressing VEGFR-3 and support the entry of cancer cells into peritumoral lymphatics. Furthermore, loss of Prox 1 function may be a driving force behind pancreatic carcinoma progression.
\end{abstract}

\section{Introduction}

Ductal adenocarcinoma of the pancreas is a devastating disease with an extremely poor prognosis and a 5-year survival rate

Correspondence to: Dr Helmut Friess, Department of General Surgery, University of Heidelberg, Im Neuenheimer Feld 110, D69120 Heidelberg, Germany

E-mail: helmut_friess@med.uni-heidelberg.de

Key words: lymphangiogenesis, Prox1, VEGF-C, pancreatic cancer, lymphatic spread below $1 \%$, making it the fourth most common cause of cancer related deaths in Western Europe $(1,2)$. Although large multicenter trials are underway and beneficial effects of adjuvant chemotherapy in patients with resected pancreatic cancer have recently been reported (3), surgical resection is the only possible option for curative treatment. However, complete resection is only possible in a minority (15-20\%) of patients and the risk of tumor relapse is high. This is attributable to rapid local growth of this aggressive cancer and early involvement of peripancreatic and para-aortal lymph nodes, which is associated with a poor prognosis $(4,5)$.

In striking contrast to the clinical implications of lymphogenic metastasis, lymphatic vessels in human pancreatic cancer have not been studied to date. In general, the contribution of tumor-associated lymphatic vessels to metastatic spread is discussed controversially $(6,7)$ and it is still not clear whether tumors establish routes for metastasis by inducing de novo formation of lymphatic vessels (lymphangiogenesis) in intratumoral and peritumoral regions. Only recently have lymphatic endothelial markers become applicable in approaching this important question. The hyaluronan receptor, LYVE-1 $(8,9)$, and the homeobox transcription factor, Prox1 $(10,11)$, display the best specificity for lymphatic endothelial cells. LYVE-1, however, is also expressed by liver sinusoidal endothelial cells (12) and Prox 1 is expressed in various nonendothelial cell types during embryogenesis, including hepatocytes, pancreatic epithelium and the developing lens $(13,14)$. As shown in this study, adult human pancreatic parenchymal cells are likewise Prox1-positive. Therefore, combination of nuclear Prox 1 staining with PECAM/CD31, a membrane marker of endothelial cells, is required for the detection of lymphatic endothelial cells in adult tissue (13).

Our current understanding of how lymphangiogenic factors orchestrate the rather complex process of lymphangiogenesis is based on studies of embryonic development. VEGF-C, a member of the vascular endothelial growth factor (VEGF) family, has been described as the first specific lymphangiogenic growth factor $(15,16)$. Its key function as a paracrine factor essential for lymphangiogenesis has recently been highlighted by studies in mice deficient in VEGF-C (17). The lymphoproliferative effect of VEGF-C is mediated by high affinity binding to the endothelial-specific receptor tyrosine kinase, VEGFR-3/flt4 $(18,19)$. Unlike VEGFR-2/flk1, a major regulator of angiogenesis, VEGFR-3 becomes restricted to lymphatic endothelial cells during embryonic development 
(20). Prox 1 is required for the initial steps of endothelial cell fate determination to the lymphatic lineage $(10,21)$, a process termed lymphatic commitment $(22,23)$, and transgenic mice deficient in Prox 1 show an arrest of lymphangiogenesis at the earliest stages (24). Additionally, Prox 1 is an essential regulator of liver and pancreas development in the foregut endoderm (25). Its possible involvement in pancreatic carcinogenesis, however, has not been studied yet.

Here, we report on the role of lymphatic vessels in pancreatic cancer progression. First, based on a morphological analysis of lymphatic vessels in pancreatic tumors and adjacent peritumoral stroma, we show that hyperplastic peritumoral lymphatics play a predominant role in lymphogenic spread of tumor cells, whereas intratumoral lymphatics are rare and mostly non-functional. Secondly, we present an expression analysis of the lymphatic growth and determination factors, VEGF-C, VEGFR-3/flt4 and Prox1, in pancreatic cancer tissue and cancer cell lines. Our results suggest that VEGF-C secreted by pancreatic carcinoma cells may act on cancer stromal cells in a paracrine fashion and induce the formation of instable and leaky vessels. Loss of Prox 1 during tumor progression points to a role for Prox 1 as a differentiation marker for pancreatic cancer cells.

\section{Materials and methods}

Human pancreatic cancer specimens. All tissue samples were obtained from patients undergoing pancreatic resection at the University Hospital of Bern and the Department of General Surgery at the University of Heidelberg. The ethical committees at the University of Bern, Switzerland, and the University of Heidelberg, Germany, approved the study protocol. Tissue specimens from 36 patients with ductal adenocarcinoma, and 30 normal human pancreatic tissue specimens were used for RNA extraction and subsequent quantitative RT-PCR and immunohistochemistry. Cancer differentiation grades (well, moderately and poorly differentiated) and UICC (Union Internationale Contre le Cancer) stages 1-4 were equally distributed among cancer tissue samples. Normal control specimens were obtained through an organ donor program. The tissue samples were snap frozen in liquid nitrogen immediately after resection and stored at $-80^{\circ} \mathrm{C}$ until further analysis was performed.

Analysis of lymphatic vessel densities was performed on intratumoral and peritumoral tissue blocks derived from specimens of clinically advanced tumors with positive lymph node status (pT3N1 according to UICC).

Cell culture experiments. Seven human pancreatic cancer cell lines (Capan-1, ASPC-1, BxPc-3, Colo-357, T3M4, Mia-PaCa-2 and Panc-1) with different grades of cellular differentiation (26) were used to study the expression of lymphangiogenic markers. The embryonic kidney cell line 293 T was used as a positive control for Prox 1 expression. Cell lines were purchased from the American Tissue Type Culture Collection (ATCC, Rockville, MD, USA) or obtained from Murray Korc (University of California, Irvine, CA, USA), and grown in RPMI-1640 medium supplemented with $10 \%$ fetal bovine serum and penicillin/streptomycin $(100 \mathrm{U} / \mathrm{ml}, 100 \mu \mathrm{g} / \mathrm{ml})(27,28)$.
RNA extraction and quantitative RT-PCR (QRT-PCR). Preparation of mRNA and cDNA, and QRT-PCR were performed as described previously (29). In brief, mRNA was isolated from cells and tissue using MagNA Pure mRNA extraction kit I (for cells) or II (for tissue) (Roche). cDNA was synthesized from mRNA by reverse transcription using a cDNA kit (First Strand cDNA Synthesis Kit, Roche) according to the manufacturer's instructions. QRT-PCR was performed with the LightCycler FastStart DNA SYBR Green kit (Roche). The primer sets for Prox1, flt-4, VEGF-C and constitutively expressed housekeeping genes, hypoxanthine phosphoribosyl transferase (HPRT) and cyclophillin B, were obtained from Search-LC GmbH (Heidelberg, Germany). The copy number of the specific transcripts was normalized to the values obtained for the housekeeping genes and expressed as number pro $\mu 1$ of input cDNA.

Antibodies. The monoclonal mouse anti-human CD31/PECAM (Dako, Hamburg, Germany, dilution 1:200) and polyclonal rabbit anti-human Prox1 (Reliatech, Braunschweig, Germany, dilution $1: 100$ ) antibodies were applied on consecutive cryosections to detect lymphatic vessels in pancreatic cancer specimens. The LYVE-1 monoclonal mouse antibody (9) was a generous gift from David G. Jackson, Oxford, UK. The VEGF-C and flt4/VEGFR-3 antibodies (goat polyclonal, Santa Cruz Biotechnologiy, Heidelberg, Germany) were used on paraffin sections at dilutions of 1:100 and 1:200, respectively. Peroxidase-conjugated goat anti-mouse and biotinylated rabbit anti-goat antibodies (Dako) were used as secondary antibodies in immunohistochemical procedures. The Prox 1 antibody was detected by immunofluorescence with a Cy3-conjugated goat anti-rabbit secondary antibody (Dianova, Hamburg, Germany).

Immunohistochemistry and immunofluorescence studies. VEGF-C and flt4/VEGFR-3 stainings were performed on paraffin sections $(8 \mu \mathrm{m})$ of formalin-fixed tissue. After deparaffinization and rehydration, antigen retrieval was performed by cooking in $0.1 \mathrm{~mol} / \mathrm{l}$ sodium citrate buffer for 25 min, followed by treatment with $3 \%$ hydrogen peroxide solution to block endogenous peroxidase activity. Incubation with primary antibodies was carried out over night at $4^{\circ} \mathrm{C}$. After standard washing steps, slides were subsequently incubated with a biotinylated secondary antibody and streptavidinperoxidase. Slides were exposed to DAB (3.3'-diaminobenzidine tetrahydrochloride, Dako) for color development and counterstained with hematoxylin.

CD31/PECAM-1 and LYVE-1 immunostainings were carried out on consecutive cryosections $(12 \mu \mathrm{m})$ of snapfrozen tissue blocks. Resulting slides were air dried over night and acetone-fixed. The staining protocol was similar to that described above, but antigen retrieval steps were skipped on cryosections. The Dako ${ }^{+}$Envision kit was used for color development.

For Prox 1 immunofluorescence studies, cryosections remained unfixed. Staining was carried out as described previously (13). The primary antibody was incubated with the sections for $1 \mathrm{~h}$ and replaced by rabbit IgG in control sections.

Assessment of lymphatic vascular densities. The mean lymphatic vascular density was assessed in intratumoral 


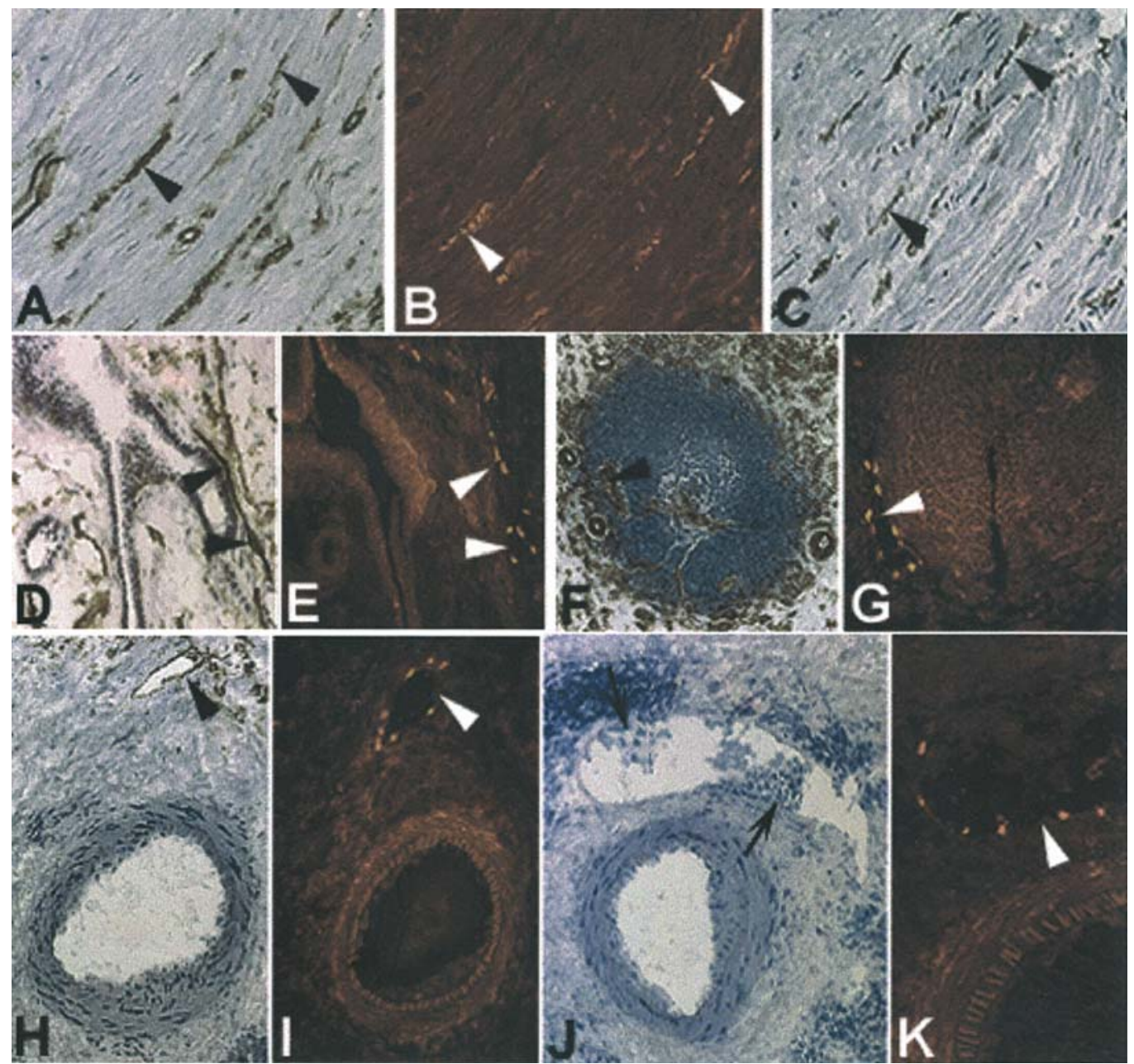

Figure 1. Morphology of lymphatic vessels in pancreatic ductal adenocarcinoma. Immunostainings for the panendothelial marker, CD31 (A, D, F and H), and the lymphatic endothelial markers, Prox 1 (B, E, G, I and K) and LYVE-1 (C). A-G, intratumoral lymphatic vessels. Lymphatic clefts (arrowheads) in the tumor stroma (A-C) and adjacent to ductal-like cancer cells (D and E) are not lumenized. F and G, lymphatic vessel (arrowhead) adjacent to an intratumoral lymphocellular infiltrate. H-K, peritumoral lymphatic vessels are located adjacent to blood vessels and display wide vascular lumina. The lymphatic endothelium (arrowhead) stains positive for CD31 (H) and Prox1 (I). J and K, tumor cells (arrows) invade an enlarged peritumoral lymphatic vessel lined by Prox1-positive lymphatic endothelium (arrowhead).

$(\mathrm{n}=15)$ and peritumoral (tumor-adjacent stroma, $\mathrm{n}=15)$ tissue blocks using CD31/PECAM-1 and Prox1 labeling of lymphatic endothelial cells on 4 different transverse sections per specimen. All vessels containing two or more Prox1positive nuclei and co-expressing CD31/PECAM-1 were counted and their number divided by the area of the optical field to obtain the vascular density per $\mathrm{mm}^{2}$. As a parameter for lymphatic vessel size, the number of endothelial cells per lymphatic vessel was determined by dividing the count of Prox1-positive endothelial cells by the number of lymphatic vessels. The classical hot-spot method for determining vascular densities could not be applied because all specimens were devoid of lymphatic vascular hot-spots.

Statistical analysis. Results are expressed as mean \pm SEM. Statistical significance of lymphatic vascular densities and mRNA expressions was determined using the unpaired t-test with Welch correlation, with a two-tailed $\mathrm{p}<0.05$ considered to be statistically significant. Linear regression analysis and Cox-regression analysis were used to assess the correlation of Prox 1 gene expression with patient survival.

\section{Results}

Morphology of lymphatic vessels in primary pancreatic carcinomas and metastases. To address the question of whether intratumoral lymphatic vessels contribute to lymphovascular spread of pancreatic carcinoma, resection specimens were screened for the existence of intratumoral lymphatic vessels using a double-staining method for the panendothelial marker, CD31, and the lymphatic endothelial marker, Prox1 (13) (Fig. 1). Parallel staining for the lymphatic endothelial marker, LYVE-1, was applied to confirm the lymphatic origin of Prox1/CD31 double-positive vessels (Fig. 1C). Intratumoral lymphatics were frequently collapsed and proper endovascular lumen formation was rare (Fig. 1A and D). In contrast to peritumoral regions, lymphatic vessels did not accompany normal blood vessels but were scattered in the tumor stroma (Fig. 1A and B). Lymphocyte infiltrates were frequently surrounded by Prox1/CD31 double-positive lymphatic vessels (Fig. 1F and G). In some specimens, collapsed lymphatic clefts could also be detected in close vicinity to cancer ducts (Fig. 1D and E). 

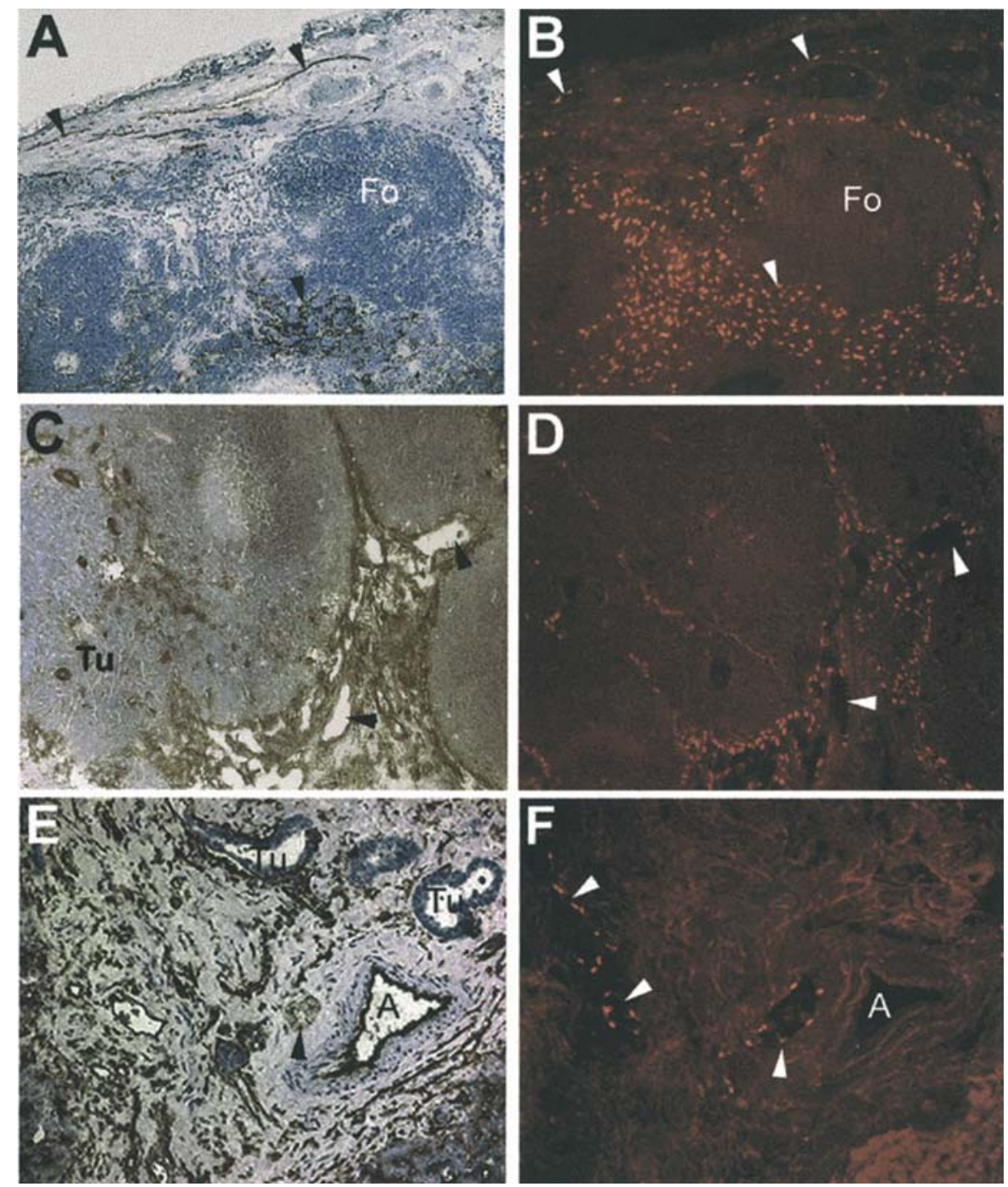

Figure 2. Lymphatic vessels in metastases from pancreatic carcinoma. Immunostainings for LYVE-1 (A), Prox1 (B, D and F) and CD31 (C and E). A and B, normal peripancreatic lymph node. Subcapsular and peritrabecular lymphatic sinuses (arrowheads) express LYVE-1 and Prox1. Fo, lymph follicle. C and D, metastatic lymph node. CD31/Prox1-positive peritrabecular lymphatic vessels (arrowheads) are dilated. E and F, hepatic metastasis. Dilated lymphatic vessels (arrowheads) are located adjacent to metastasized tumor ducts (Tu). A, artery.

The morphology of lymphatic vessels in the peritumoral tissue was strikingly different from that of intratumoral lymphatics. Almost all lymphatic vessels in the peritumoral area had wide endovascular lumina (Fig. 1H-K). Unlike their intratumoral counterparts, peritumoral lymphatics joined the blood vascular routes. The invasion of tumor cells into engorged peritumoral lymphatic vessels could be detected in several specimens (Fig. $1 \mathrm{~J}$ and $\mathrm{K}$ ).

Next, we examined the morphology of the lymphatic vasculature in peripancreatic lymph nodes. Subcapsular sinuses in the cortex expressed the lymphendothelial markers, LYVE-1 and Prox1 (Fig. 2A and B). Marked Prox1 staining was present in sinuses surrounding the lymph follicles, but no Prox1-positive cells could be detected within the lymph follicles and germinal centers of tumor-free lymph nodes (Fig. 2B). In metastatic lymph nodes, however, peritrabecular lymphatic sinuses were dilated and Prox1/CD31-positive lymphatic vessels were present within the metastasized tumor mass (Fig. 2C and D).

Moreover, five liver metastases of pancreatic ductal adenocarcinomas were screened for the existence of lymphatic vessels. In all cases, lymphatic vessels were present in close proximity to metastasized tumor cells (Fig. 2E and F).

Lymphatic vessel densities in intra- and peritumoral regions of pancreatic carcinoma. To assess the contribution of tumorrelated lymphatic vessels to metastatic spread, lymphatic vessel densities were analyzed in representative intratumor and peritumor tissue specimens from patients with ductal adenocarcinoma of clinically advanced stages, including a positive lymph node status (all tumors, stage pT3N1).

The average lymphatic vessel density (Prox 1/CD31 double-positive vessels per area) was 1.3 and 2.4 vessels/ $\mathrm{mm}^{2}$ in intra- and peritumoral regions, respectively (Fig. 3, left panel), which is much lower than the lymphatic vessel density reported in other tumor entities $(30,31)$ and pancreatic endocrine tumors (32). Moreover, intratumoral lymphatic 'hot-spots' with proliferating lymphatic vessels, which have been reported to correlate with metastasis and clinical outcome in other tumors $(30,31,33,34)$, were not observed in any of the analyzed pancreatic carcinomas. The average lymphatic vessel density in peritumoral tissue was 1.8 -fold higher than 

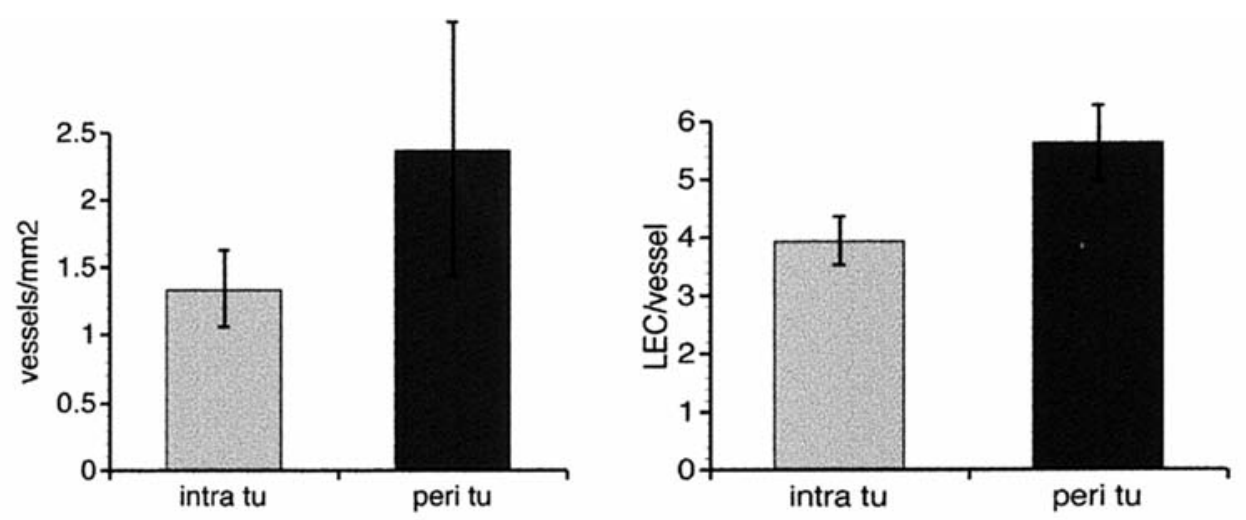

Figure 3. Lymphatic vessel density (left) is low in both intra- and peritumoral regions of metastatic ductal adenocarcinoma. Right, lymphatic endothelial cell count per lymphatic vessel. Peritumoral lymphatic vessels contain significantly more endothelial cells than intratumoral lymphatics. Data are presented as mean \pm SEM, $\mathrm{p}<0.05$ in right panel.
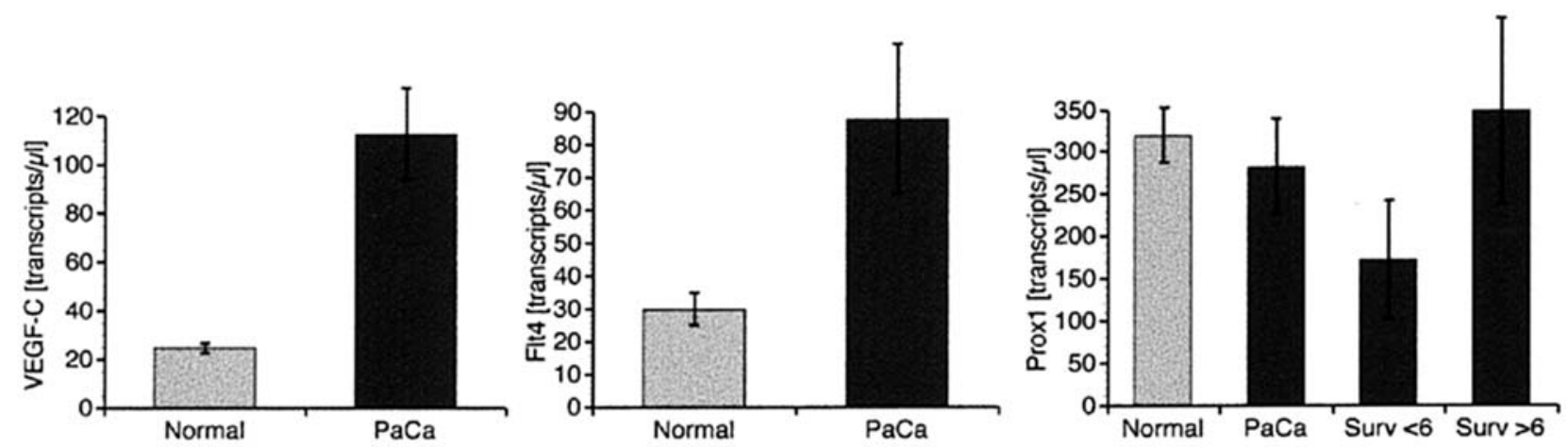

Figure 4. Quantitative RT-PCR data for VEGF-C, VEGFR-3/flt4 and Prox1 in pancreatic carcinoma (PaCa) and normal control specimens. Left and middle, VEGF-C and VEGFR-3/flt4 mRNA-levels are significantly higher in PaCa than in normal pancreas. Right, Prox1 is expressed in PaCa and normal pancreas. Carcinomas from patients with survival below 6 months (Surv <6) show lower Prox 1 mRNA levels than those from patients with longer survival. Mean \pm SEM of transcripts $/ \mu 1$ of input cDNA, $\mathrm{p}<0.001$ in left panel, $\mathrm{p}<0.05$ in middle panel.

in intratumoral regions, but this difference lacked statistical significance.

To obtain additional information about the average size of lymphatic vessels in intra- and peritumoral regions, we determined the number of endothelial cells per lymphatic vessel (Prox1-positive nuclei per CD31-positive vessel; Fig. 3, right panel). On average, sections of peritumoral lymphatic vessels contained significantly more endothelial cells than sections of intratumoral lymphatics (5.7 vs. 3.9 respectively, $\mathrm{p}<0.05)$, properly reflecting the morphological differences reported above (Fig. 1).

On the background of this morphological and morphometric analysis we conclude that extensive lymphangiogenesis is absent in both intra- and peritumoral regions. Whereas lymphatics within the tumor remain widely nonfunctional, engorged peritumoral lymphatic vessels are key structures facilitating the lymphangiogenic metastasis of pancreatic cancer.

Expression of VEGF-C, VEGFR-3 and Proxl in pancreatic tissue. To evaluate whether factors secreted by tumor cells promote lymphogenic metastasis of pancreatic cancer, we analyzed the expression of the lymphangiogenic growth factor, VEGF-C; its receptor, VEGFR-3; and transcription factor, Prox1, in normal exocrine pancreas ( $\mathrm{n}=30$ tissue specimens) and ductal adenocarcinoma of the pancreas $(n=36$ tissue specimens). QRT-PCR was performed for quantitative analysis of gene expression profiles. Spatial expression patterns were investigated using immunohistochemical procedures.

mRNA expression levels of lymphangiogenic growth factor, $V E G F-C$, were significantly $(\mathrm{p}<0.001)$ increased in pancreatic cancer specimens compared to normal tissue samples (Fig. 4, left panel). $V E G F-C$ protein was expressed in the cytoplasm of ductal-like cancer cells (Fig. 5A). Acini and ducts of the normal exocrine pancreas did not show VEGF-C immunoreactivity. Likewise, mRNA expression levels of VEGFR-3/flt4, the receptor tyrosine kinase mediating VEGF-C signaling, were significantly $(\mathrm{p}<0.05)$ up-regulated in pancreatic cancer specimens (Fig. 4, middle panel). Consistently, immunohistochemical analysis revealed VEGFR-3/flt4protein expression in pancreatic cancer stroma cells and microvessels (Fig. 5B). VEGFR-3/flt4-positive microvessels did not co-express the lymphatic markers, Prox 1 and LYVE. Importantly, neither $V E G F-C$ nor $V E G F R$-3/flt4 gene expression in pancreatic carcinoma correlated with patient survival (linear regression analysis, correlation coefficient $\mathrm{r}=-0.23, \mathrm{p}=0.45$ for $V E G F-C ; \mathrm{r}=-0.4, \mathrm{p}=0.24$ for $V E G F R-3)$.

Proxl, which is a marker of both lymphatic endothelial cells and the embryonic pancreas, was highly expressed in normal pancreas tissue and slightly down-regulated in pancreatic 


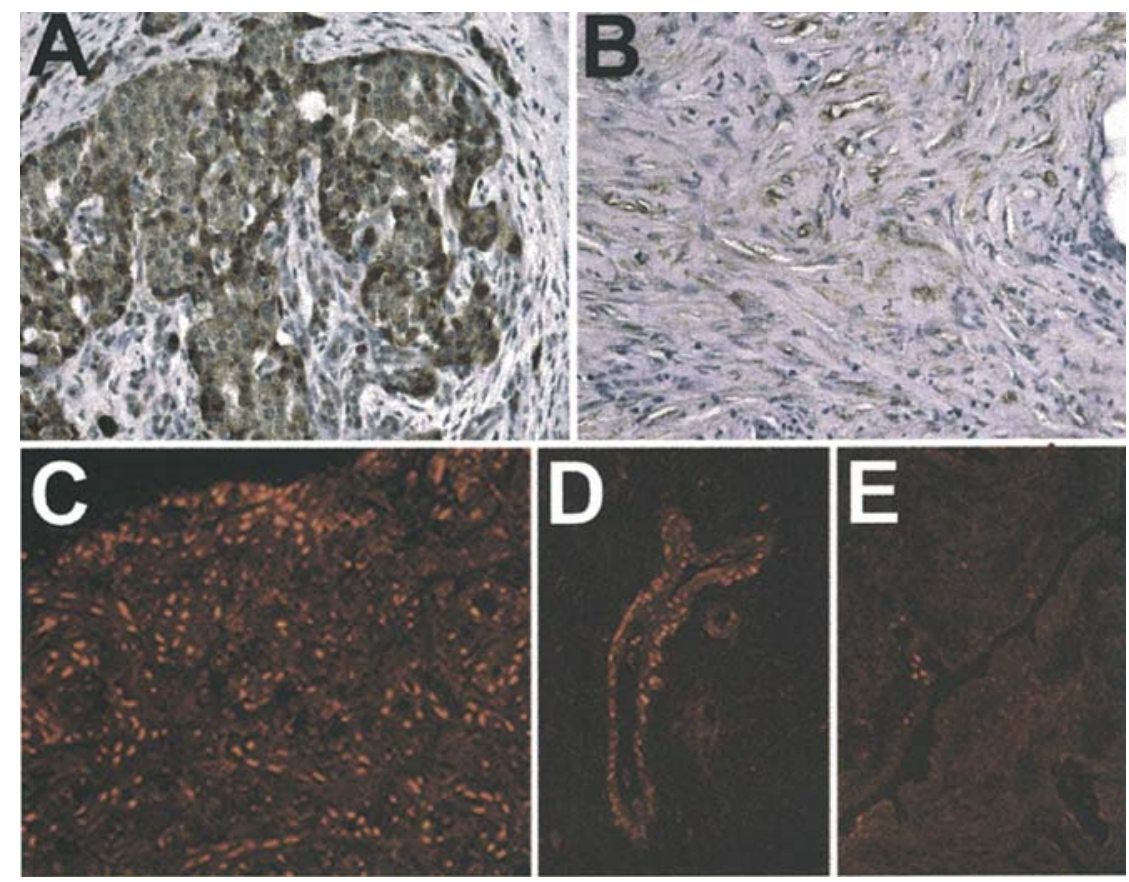

Figure 5. A, pancreatic carcinoma, representative immunostaining for VEGF-C. Cancer cells show VEGF-C immunoreactivity (arrows). B, immunohistochemistry of VEGFR-3/flt4 in pancreatic cancer stroma. Multiple stroma cells and microvessels are VEGFRE-3 positive. C-E, Prox1 immunostaining of pancreatic tissue. In normal pancreas, nuclei of acini and ducts are Prox1 positive (C). In ductal adenocarcinoma, some cancer ducts show Prox-1-positive nuclei (D), whereas others are devoid of Prox 1 immunofluorescence (E).

carcinoma (Fig. 4, right panel). Immunoflourescence revealed nuclear Prox 1 protein expression in acini and ducts of normal pancreas specimens (Fig. 5C). In some pancreatic adenocarcinomas, Proxl was expressed in cancer ducts. Other pancreatic carcinoma specimens, however, were completely devoid of Proxl immunofluorescence or showed expression only in a minority of cancer cells (Fig. 5D and E). Strikingly, mean Proxl gene expression was lower in a subgroup of cancer specimens derived from patients with a survival less than 6 months after diagnosis than in pancreatic carcinomas from patients with longer survival (Fig. 4, right panel). Linear regression analysis revealed a correlation of Proxl expression in pancreatic carcinoma with longer patient survival (correlation coefficient $\mathrm{r}=0.6, \mathrm{p}=0.015$; borderline significance, $\mathrm{p}=0.086$, in Cox regression analysis).

Expression of VEGF-C, VEGFR-3 and Proxl in pancreatic cancer cell lines. To complete the expression analysis of lymphangiogenic determinants in pancreatic cancer, we performed in vitro expression profiling of VEGF-C, VEGFR-3 and Prox in different pancreatic ductal carcinoma cell lines (Capan-1, Colo-357, ASPC-1, BxPc-3 Panc-1, Mia PaCa-2, T3M4).

Quantitative RT-PCR revealed the presence of VEGF-C mRNA transcripts in five out of seven cell lines (T3M4: 1381+251 transcripts/ $\mu$ l cDNA; Mia PaCa-2: 930+97; Panc-1: 323+97; Colo: 225+19; BxPc: $28+7$ ). Low expression of VEGFR-3/flt4 mRNA could be detected in the poorly differentiated Panc- 1 cell line (9+5 transcripts/ $\mu 1 \mathrm{cDNA})$; all other tested cell lines were devoid of VEGFR-3/flt4 expression.

Prox 1 mRNA was detectable in three out of seven tested pancreatic cancer cell lines. Interestingly, Proxl expression was low or absent in those cell lines displaying a low grade of cellular differentiation (26) (Mia PaCa-2 and Panc-1: undetectable; T3M4: 2+1 transcripts/ $\mu 1$ cDNA). In contrast, the well-differentiated Capan-1 cell line and the moderately differentiated ASPC-1 cell line expressed highest Proxl mRNA levels (Capan-1: 17+5 transcripts/ $\mu$ l cDNA; ASPC-1: $26+9)$. The embryonic kidney cell line $293 \mathrm{~T}$, used as a positive control in this experiment, showed high Proxl mRNA levels $(217+36$ transcripts/ $\mu 1 \mathrm{cDNA})$.

\section{Discussion}

Peritumoral lymphatics promote pancreatic cancer cell dissemination. Several recent studies addressed the role of lymphangiogenesis in metastatic spread of solid tumors but led to rather heterogeneous results. The presence of intratumoral lymphatic vessels and de novo formation of lymphatic vessels have been documented in some malignancies $(30,33,35)$ and intratumoral lymphatic vessels have been reported to correlate with a higher risk of local relapse and poor prognosis (34). Other morphological studies demonstrated a lack of intratumoral lymphatic vessels in malignant tumors $(36,37)$. Based on experimental data obtained from animal models, there is growing evidence that peritumoral lymphatic vessels promote tumor cell dissemination via the lymphatic system, that this process occurs independently of intratumoral lymphatics (38), and that lymphatic vessels within solid tumors remain non-functional despite the presence of lymphangiogenic growth factor, VEGF-C (39). The present study of lymphatic vessels in human pancreatic cancer provides patient-derived data to support these observations, as it documents the occurrence of enlarged peritumoral lymphatics in metastatic cancer specimens. Importantly, the intratumoral lymphatic vascular density is low even in metastasized pancreatic cancer 
and lack of lumen formation is a common feature of intratumoral lymphatics, altogether suggesting a predominant role of peritumoral lymphatics in metastatic spread. However, and differently from previously reported findings in skin cancer (33), we could not detect signs of active lymphangiogenesis in peritumoral regions. In fact, tumor cells preferentially invaded peritumoral lymphatics that could be identified as pre-existing lymphatic vessels by their position adjacent to normal blood vessels. It thus seems likely that invasion of pre-existing peritumoral lymphatic vessels is the crucial step for the initiation of lymphatic vessel invasion by tumor cells.

Role of VEGF-C and VEGFR-3 in pancreatic carcinoma. The search for molecular mechanisms facilitating lymphovascular tumor spread focuses on factors which induce sprouting or enlargement of lymphatic vessels at the tumor margin or within the tumor itself. A likely candidate is VEGF-C, the first specific lymphotrophic growth factor detected $(15,16,18)$. VEGF-C overexpression has been observed in various malignancies $(7,40,41)$. We found that VEGF-C was significantly up-regulated in human pancreatic carcinoma tissue and in pancreatic cancer cell lines, suggesting that pancreatic parenchymal cells are capable of switching on VEGF-C-expression upon transition to a malignant phenotype. These results underline the possible role of VEGF-C in pancreatic cancer progression and metastasis. However, as pancreatic tumors are devoid of active lymphangiogenesis, VEGF-C does not seem to promote the formation of lymph node metastases by inducing a lymphangiogenic switch, comparable to the angiogenic switch described by Folkman (42). It appears more likely that tumor-derived VEGF-C facilitates lymphatic vessel invasion by cancer cells, an event mainly taking place in the tumor periphery. Indeed, when transgenic mice are engineered to overexpress VEGF-C in endocrine tumors of the pancreas (43), these tumors are surrounded by well-developed lymphatics in the absence of intratumoral lymphangiogenesis and frequently produce pancreatic lymph node metastases.

VEGF-C has both angiogenic and lymphangiogenic properties, as it binds to the angiogenic receptor, VEGFR-2/ flk1, and the lymphendothelial-specific receptor, VEGFR-3/ flt4 (18). Activity towards VEGFR-3/flt4 increases upon proteolytic processing of the VEGF-C propeptide, and the fully processed VEGF-C binds and activates VEGFR-2/flk1 (19). Here, we show that VEGFR-3/flt4, unlike VEGFR-2/flk1 (28), is not expressed at relevant levels in pancreatic cancer cells. Increased VEGFR-3/flt4-levels that were present in pancreatic cancer tissue seem to stem from marked expression in the cancer stroma. Multiple intratumoral microvessels stained positive for VEGFR-3/flt4 but were devoid of lymphoendothelial markers. Therefore, in addition to increasing vascular permeability, thereby facilitating lymphogenic metastasis, VEGF-C secreted by pancreatic cancer cells seems to be involved in the paracrine signaling network inducing angiogenic growth in pancreatic cancer. A similar effect of VEGF-C on tumor angiogenesis exists in breast cancer, where VEGF-C secreted by the intraductal carcinoma cells acts predominantly as an angiogenic growth factor for blood vessels (44). It appears paradoxical, however, that VEGF-C expression does not correlate with patient survival in pancreatic cancer, despite the fact that it correlates positively with lymphatic vessel invasion and lymph node metastasis (45). The reason might be that excess production of pro-angiogenic cytokines (such as VEGF and VEGF-C) in the absence of vascular stabilization factors (such as angiopoietin-1) results in the formation of leaky and fragile vessels, rather than establishing a functional blood vascular network (46).

Thus, VEGF-C secretion by cancer cells facilitates lymph node invasion but does not necessarily effect the outcome of the disease.

Role for Proxl in pancreatic cancer progression. The present study reveals insights into the expression pattern of the transcription factor, Prox 1, in pancreatic cancer. Cloned as the homologue of the Drosophila homeobox gene, prospero $(11,47)$, Prox 1 expression has been reported in the development of organ systems as different as the eye (48), taste buds (49), CNS and cardiomyocytes (14). Prox 1 has likewise been assigned a major role in the developing epithelial anlagen of the liver and the pancreas $(25,50)$. Additionally, Prox 1 is essential for the embryonic development of lymphatic vessels. Prox1-null mice have normal blood vessels but do not develop lymphatics (24). Prox 1 expression in the adult pancreas has not been studied to date. We have observed strong and homogenous Prox 1 expression in parenchymal cells of the normal exocrine pancreas, whereas the endocrine cells are negative. Strikingly, Prox 1 expression appeared to be downregulated in pancreatic cancer tissue. This finding is clinically relevant, as, in this study, loss of Prox 1 gene function was accompanied by poorer patient survival. It is further remarkable that Prox 1 was expressed by cell lines of higher cellular differentiation grades but absent in poorly differentiated pancreatic cancer cell lines. Altogether, Prox 1 emerges as a possible new differentiation marker that could be of practical use in histopathological grading of pancreatic cancer tissue and as a prognostic factor, warranting further evaluation.

In conclusion, this study provides first insights into the significance of tumor-associated lymphatic vessels in lymphogenic spread of human pancreatic cancer, suggesting that preexisting peritumoral lymphatic spaces play a predominant role in promoting cancer cell dissemination via the lymphatic system. Moreover, the presented expression analysis of lymphatic factors suggests a role for Prox 1 in the cellular dedifferentiation process. Prox 1 expression was studied in adult human pancreatic tissue for the first time and was shown to be down-regulated in pancreatic cancer, pointing to the potential prognostic value of Prox 1 gene expression levels in pancreatic cancer. In contrast to Prox 1, VEGF-C was up-regulated in pancreatic cancer and VEGF-C/VEGFR-3 interactions seem to be involved in the paracrine signaling network mediating angiogenesis and vascular permeability.

\section{Acknowledgements}

This study was supported by grants from the Deutsche Forschungsgemeinschaft (SFB 402, D4; SCHN 947/1-1). We are grateful to Professor David G. Jackson, Oxford, UK, for providing the LYVE-1 antibody. We also thank Mrs Monika Meinhardt, Mrs Brunhilde Benzinger, Mrs Elvira Mohr and Mrs Meta Stadter for their excellent technical assistance. 


\section{References}

1. Neoptolemos JP, Dunn JA, Stocken DD, et al: Adjuvant chemoradiotherapy and chemotherapy in resectable pancreatic cancer: a randomised controlled trial. Lancet 358: 1576-1585, 2001.

2. Parker SL, Davis KJ, Wingo PA, Ries LA and Heath CW Jr: Cancer statistics by race and ethnicity. CA Cancer J Clin 48: $31-48,1998$.

3. Neoptolemos JP, Stocken DD, Friess H, et al: A randomized trial of chemoradiotherapy and chemotherapy after resection of pancreatic cancer. N Engl J Med 350: 1200-1210, 2004.

4. Ridwelski K, Meyer F, Ebert M, Malfertheiner P and Lippert $\mathrm{H}$ : Prognostic parameters determining survival in pancreatic carcinoma and, in particular, after palliative treatment. Dig Dis 19: 85-92, 2001

5. Johnstone PA and Sindelar WF: Lymph node involvement and pancreatic resection: correlation with prognosis and local disease control in a clinical trial. Pancreas 8: 535-539, 1993.

6. Jain RK and Fenton BT: Intratumoral lymphatic vessels: a case of mistaken identity or malfunction? J Natl Cancer Inst 94: 417-421, 2002

7. Pepper MS: Lymphangiogenesis and tumor metastasis: myth or reality? Clin Cancer Res 7: 462-468, 2001

8. Banerji S, Day AJ, Kahmann JD and Jackson DG: Characterization of a functional hyaluronan-binding domain from the human CD44 molecule expressed in Escherichia coli. Protein Expr Purif 14: 371-381, 1998.

9. Banerji S, Ni J, Wang SX, et al: LYVE-1, a new homologue of the CD44 glycoprotein, is a lymph-specific receptor for hyaluronan. J Cell Biol 144: 789-801, 1999.

10. Wigle JT, Harvey N, Detmar M, et al: An essential role for Prox1 in the induction of the lymphatic endothelial cell phenotype. EMBO J 21: 1505-1513, 2002

11. Oliver G, Sosa-Pineda B, Geisendorf S, Spana EP, Doe CQ and Gruss P: Prox 1, a prospero-related homeobox gene expressed during mouse development. Mech Dev 44: 3-16, 1993.

12. Mouta Carreira C, Nasser SM, Di Tomaso E, et al: LYVE-1 is not restricted to the lymph vessels: expression in normal liver blood sinusoids and down-regulation in human liver cancer and cirrhosis. Cancer Res 61: 8079-8084, 2001.

13. Wilting J, Papoutsi M, Christ B, et al: The transcription factor Prox 1 is a marker for lymphatic endothelial cells in normal and diseased human tissues. FASEB J 16: 1271-1273, 2002.

14. Rodriguez-Niedenfuhr M, Papoutsi M, Christ B, et al: Prox1 is a marker of ectodermal placodes, endodermal compartments, lymphatic endothelium and lymphangioblasts. Anat Embryol 204: 399-406, 2001

15. Oh SJ, Jeltsch MM, Birkenhager R, et al: VEGF and VEGF-C specific induction of angiogenesis and lymphangiogenesis in the differentiated avian chorioallantoic membrane. Dev Biol 188: 96-109, 1997

16. Jeltsch M, Kaipainen A, Joukov V, et al: Hyperplasia of lymphatic vessels in VEGF-C transgenic mice. Science 276: 1423-1425, 1997.

17. Karkkainen MJ, Haiko P, Sainio K, et al: Vascular endothelial growth factor $\mathrm{C}$ is required for sprouting of the first lymphatic vessels from embryonic veins. Nat Immunol 5: 74-80, 2004.

18. Joukov V, Pajusola K, Kaipainen A, et al: A novel vascular endothelial growth factor, VEGF-C, is a ligand for the Flt4 (VEGFR-3) and KDR (VEGFR-2) receptor tyrosine kinases. EMBO J 15: 290-298, 1996

19. Joukov V, Sorsa T, Kumar V, et al: Proteolytic processing regulates receptor specificity and activity of VEGF-C. EMBO J 16: 3898-3911, 1997

20. Kaipainen A, Korhonen J, Mustonen T, et al: Expression of the fms-like tyrosine kinase 4 gene becomes restricted to lymphatic endothelium during development. Proc Natl Acad Sci USA 92: 3566-3570, 1995

21. Hong YK, Harvey N, Noh YH, et al: Prox1 is a master control gene in the program specifying lymphatic endothelial cell fate. Dev Dyn 225: 351-357, 2002.

22. Alitalo K and Carmeliet P: Molecular mechanisms of lymphangiogenesis in health and disease. Cancer Cell 1: 219-227, 2002.

23. Jain RK and Padera TP: Development. Lymphatics make the break. Science 299: 209-210, 2003.

24. Wigle JT and Oliver G: Prox 1 function is required for the development of the murine lymphatic system. Cell 98: 769-778, 1999.

25. Burke $Z$ and Oliver G: Prox 1 is an early specific marker for the developing liver and pancreas in the mammalian foregut endoderm. Mech Dev 118: 147-155, 2002.
26. Sipos B, Moser S, Kalthoff H, Torok V, Lohr M and Kloppel G: A comprehensive characterization of pancreatic ductal carcinoma cell lines: towards the establishment of an in vitro research platform. Virchows Arch 442: 444-452, 2003.

27. Li J, Kleeff J, Guo J, et al: Effects of STI571 (gleevec) on pancreatic cancer cell growth. Mol Cancer 2: 32, 2003

28. Buchler P, Reber HA, Buchler MW, Friess H and Hines OJ: VEGF-RII influences the prognosis of pancreatic cancer. Ann Surg 236: 738-749, 2002.

29. Guo J, Kleeff J, Li J, et al: Expression and functional significance of CDC25B in human pancreatic ductal adenocarcinoma. Oncogene 23: 71-81, 2004.

30. Straume O, Jackson DG and Akslen LA: Independent prognostic impact of lymphatic vessel density and presence of low-grade lymphangiogenesis in cutaneous melanoma. Clin Cancer Res 9: 250-256, 2003

31. Birner P, Schindl M, Obermair A, Breitenecker G, Kowalski H and Oberhuber G: Lymphatic microvessel density as a novel prognostic factor in early-stage invasive cervical cancer. Int J Cancer 95: 29-33, 2001.

32. Sipos B, Klapper W, Kruse ML, Kalthoff H, Kerjaschki D and Kloppel G: Expression of lymphangiogenic factors and evidence of intratumoral lymphangiogenesis in pancreatic endocrine tumors. Am J Pathol 165: 1187-1197, 2004.

33. Dadras SS, Paul T, Bertoncini J, et al: Tumor lymphangiogenesis: a novel prognostic indicator for cutaneous melanoma metastasis and survival. Am J Pathol 162: 1951-1960, 2003

34. Maula SM, Luukkaa M, Grenman R, Jackson D, Jalkanen S and Ristamaki R: Intratumoral lymphatics are essential for the metastatic spread and prognosis in squamous cell carcinomas of the head and neck region. Cancer Res 63: 1920-1926, 2003

35. Yonemura Y, Fushida S, Bando E, et al: Lymphangiogenesis and the vascular endothelial growth factor receptor (VEGFR)-3 in gastric cancer. Eur J Cancer 37: 918-923, 2001

36. De Waal RM, van Altena MC, Erhard H, Weidle UH, Nooijen PT and Ruiter DJ: Lack of lymphangiogenesis in human primary cutaneous melanoma. Consequences for the mechanism of lymphatic dissemination. Am J Pathol 150: 1951-1957, 1997.

37. Williams CS, Leek RD, Robson AM, et al: Absence of lymphangiogenesis and intratumoural lymph vessels in human metastatic breast cancer. J Pathol 200: 195-206, 2003.

38. Padera TP, Kadambi A, Di Tomaso E, et al: Lymphatic metastasis in the absence of functional intratumor lymphatics. Science 296 : 1883-1886, 2002.

39. Leu AJ, Berk DA, Lymboussaki A, Alitalo K and Jain RK: Absence of functional lymphatics within a murine sarcoma: a molecular and functional evaluation. Cancer Res 60: 4324-4327, 2000

40. Salven P, Lymboussaki A, Heikkila P, et al: Vascular endothelial growth factors VEGF-B and VEGF-C are expressed in human tumors. Am J Pathol 153: 103-108, 1998.

41. Karpanen $\mathrm{T}$ and Alitalo K: Lymphatic vessels as targets of tumor therapy? J Exp Med 194: F37-F42, 2001.

42. Hanahan D and Folkman J: Patterns and emerging mechanisms of the angiogenic switch during tumorigenesis. Cell 86: 353-364, 1996

43. Mandriota SJ, Jussila L, Jeltsch M, et al: Vascular endothelial growth factor-C-mediated lymphangiogenesis promotes tumour metastasis. EMBO J 20: 672-682, 2001

44. Valtola R, Salven P, Heikkila P, et al: VEGFR-3 and its ligand VEGF-C are associated with angiogenesis in breast cancer. Am J Pathol 154: 1381-1390, 1999.

45. Tang RF, Itakura J, Aikawa T, et al: Overexpression of lymphangiogenic growth factor VEGF-C in human pancreatic cancer Pancreas 22: 285-292, 2001.

46. Carmeliet P and Jain RK: Angiogenesis in cancer and other diseases. Nature 407: 249-257, 2000

47. Zinovieva RD, Duncan MK, Johnson TR, Torres R, Polymeropoulos $\mathrm{MH}$ and Tomarev SI: Structure and chromosomal localization of the human homeobox gene Prox 1. Genomics 35: 517-522, 1996.

48. Wigle JT, Chowdhury K, Gruss P and Oliver G: Prox 1 function is crucial for mouse lens-fibre elongation. Nat Genet 21: 318-322, 1999.

49. Miura H, Kusakabe Y, Kato H, et al: Co-expression pattern of Shh with Prox1 and that of Nkx2.2 with Mash1 in mouse taste bud. Gene Expr Patterns 3: 427-430, 2003

50. Sosa-Pineda B, Wigle JT and Oliver G: Hepatocyte migration during liver development requires Prox1. Nat Genet 25: 254-255, 2000 